\title{
Prevalência de lesões microbianas bucais em usuários de prótese dentária cadastrados em uma Unidade de Saúde da Família de Jequié - Ba
}

\author{
Prevalence of microbial oral lesions in prosthesis users registered \\ in a Family Health Unit of Jequié-Ba \\ Prevalencia de lesiones microbiana orales en usuarios de prótesis dental \\ registrados en una Unidad de Salud de la Familia de Jequié-Ba \\ Mariana de Jesus SANTOS ${ }^{1}$ \\ Cláudio Leite de SANTANA ${ }^{2}$ \\ Rita de Cássia Dias Viana ANDRADE ${ }^{2}$ \\ Taís de Lima e FRAGA ${ }^{2}$ \\ Fabio Ornellas PRADO ${ }^{3}$
${ }^{I}$ Acadêmica do Curso de Odontologia, Departamento de Saúde I, Universidade Estadual do Sudoeste da Bahia, UESB, Jequié, BA, Brasil ${ }^{2}$ Professor(a) Assistente, Departamento de Saúde I, Universidade Estadual do Sudoeste da Bahia, UESB, Jequié, BA, Brasil \\ ${ }^{3}$ Professor Titular, Departamento de Saúde I, Universidade Estadual do Sudoeste da Bahia, UESB, Jequié, BA, Brasil
}

\begin{abstract}
Resumo
Objetivo: Avaliar a prevalência de lesões orais em usuários de prótese dentária. Material e método: 110 usuários de próteses dentárias foram avaliados quanto ao sexo, faixa etária, tipo de prótese, tempo de uso de prótese e tempo de uso da prótese atual, frequência e métodos de higienização bucal e da prótese, uso noturno da prótese e consumo de bebidas alcoólicas e fumo. Para coleta dos dados realizou-se o exame clínico dos participantes e posterior orientação de higiene bucal e da prótese. Resultado: 34,6\% dos usuários de prótese exibiram algum tipo de patologia, sendo a estomatite protética (27,3\%) a mais prevalente. 2,7\% dos participantes desenvolveram queilite e 4,6\% uma associação de estomatite e queilite. Não houve diferença significativa entre os sexos quanto à presença de lesão. 46,4\% dos usuários apresentavam higiene bucal deficiente e desses 54,9\% possuíam algum tipo de lesão bucal. A frequência de higienização demonstrou ser um dos fatores determinantes no processo de desenvolvimento de lesões. 71,9\% dos participantes relataram fazer uso noturno da prótese. $30 \%$ dos que bebem e 30\% dos fumantes ativos possuíam lesão bucal. Conclusão: Há ainda um grande número de pessoas acometidas por lesões orais de simples diagnóstico e intervenção. Assim, faz-se extremamente necessário a contínua orientação, conscientização e acompanhamento profissional da população para uma melhor qualidade de vida.
\end{abstract}

Descritores: Prótese Dentária; Prevalência; Mucosa Bucal; Patologia Bucal.

\begin{abstract}
Objective: To evaluate the prevalence of oral mucosal lesions in denture wearers. Material and method: 110 denture wearers were evaluated according to sex, age, type of denture, length of denture use and length of current denture use, frequency and methods of oral and denture cleaning, overnight denture wearing and alcohol consumption and smoking habits. For data collection a clinical examination of the participants and a posterior oral and denture hygiene orientation was performed. Results: $34.6 \%$ of the wearers exhibited some king of pathology, being Prosthetic Stomatitis the most common lesion (27.3\%). 2.7\% of the participants developed cheilitis and $4.6 \%$ an association of stomatitis and cheilitis. There was no significant difference between sexes regarding presence of lesion. $46.4 \%$ of the wearers had poor oral hygiene and $54.9 \%$ of those had some type of oral lesion. Frequency of cleaning proved to be one of the determinant factors in the process of development of lesions. $71.9 \%$ of the participants reported overnight denture wearing. 30\% of those who drink and $30 \%$ of the smokers had oral lesion. Conclusion: There are still a large number of people suffering from oral lesion of simple diagnosis and intervention. Therefore, it is extremely necessary the continuous orientation, awareness and professional support of the population for a better quality of life.
\end{abstract}

Descriptors: Dental Prosthesis; Prevalence; Mouth Mucosa; Pathology, Oral.

\section{Resumen}

Objetivo: evaluar la prevalencia de lesiones orales en prótesis dental. Material y método: se evaluaron 110 usuarios de prótesis dentales en cuanto a sexo, edad, tipo de prótesis, prótesis y uso tiempo tiempo actual prótesis, frecuencia y métodos de higiene oral y la prótesis, noche de la prótesis y el consumo de bebidas alcohólicas y tabaco. Recolección de datos llevó a cabo si el examen clínico de los participantes e higiene oral posterior y orientación de la prótesis. Resultado: 34.6\% de los usuarios de prótesis exhibieron algún tipo de patología, siendo la prótesis estomatitis $(27,3 \%)$ es la más frecuente. $2.7 \%$ de los participantes desarrolló queilitis y $4.6 \%$ una estomatitis y queilitis. No hubo ninguna diferencia significativa entre los sexos con respecto a la presencia de lesiones. $46,4 \%$ de los usuarios tenían mala higiene bucal y 54.9\% tenía algún tipo de lesión. La frecuencia de limpieza demostró para ser uno de los factores determinantes en el desarrollo de las lesiones. $71,9 \%$ de los participantes registrados haciendo uso nocturno de la prótesis. 30\% de los que beben y el $30 \%$ de los fumadores activos tenían lesión oral. CONCLUSIÓN: todavía hay un gran número de personas afectadas por lesiones orales de diagnóstico simple y de intervención. Por lo tanto, es extremadamente necesario el curso orientación, conocimiento y supervisión profesional de la población para una mejor calidad de vida.

Descriptores: Prótesis Dental; Prevalencia; Mucosa Bucal; Patología Bucal. 


\section{INTRODUÇÃO}

A perda permanente do elemento dentário é um acometimento patológico da cavidade bucal frequentemente causando um impacto físico e psicossocial muitas vezes negativo. A limitação da função mastigatória e o desenvolvimento de baixa estima, por exemplo, afeta diretamente a qualidade de vida de um indivíduo. A reconstituição protética dos elementos perdidos tem o intuito de devolver função, estética e bem-estar ao paciente. Porém, a eficácia desse tipo de tratamento requer manutenção contínua e comprometimento por parte do usuário a fim de evitar problemas futuros como lesões orais de tecido mole, cáries e doenças periodontais ${ }^{1}$.

A perda dentária compromete a mastigação, escolha dos alimentos e o processo digestório, além de causar alteração na fala e estética facial ${ }^{2}$. A reposição protética dos dentes perdidos ou a substituição de peças antigas envolve um processo de adaptação tanto das próteses quanto mudanças na cavidade bucal como por exemplo alteração do paladar e redução do fluxo salivar ${ }^{3}$. Adicionalmente, essas alterações podem estar ligadas ao uso de medicamentos como antihipertensivos, ansiolíticos, antidepressivos e terapias para tratamento de câncer relacionadas ou não à idade do paciente ${ }^{4}$.

$\mathrm{O}$ aparecimento de lesões orais relacionado ao uso de próteses dentárias parciais ou totais, removíveis ou não, possui etiologia multifatorial. Fatores como colonização maciça de microrganismos nas próteses, principalmente por fungos do gênero Candida, falhas no planejamento e na confecção desses aparatos, má higienização, falta de coordenação motora do paciente, traumas mecânicos, idade da prótese, uso contínuo da mesma, entre outros estão intimamente relacionados com o desenvolvimento de lesões causadas por microrganismos ${ }^{5,6}$.

O fungo Candida albicans é o principal agente causador de muitas dessas lesões como por exemplo o desenvolvimento de estomatite por prótese, candidíase, leucoplasia por Cândida e queilite angular?

Essas lesões, muitas vezes, são assintomáticas, porém, nos casos sintomáticos os pacientes podem apresentar quadros de dor, prurido, distúrbios salivares, sensação de queimação e inflamação, circunscrita ou difusa, da mucosa que é coberta pelo aparato ${ }^{5,8}$.

O tratamento para essas lesões propõe desde a higienização da cavidade bucal e das próteses, bem como a substituição de próteses antigas ou inadequadas ${ }^{8}$, até o emprego de medicamentos como o miconazol, a nistatina e outros agentes antifúngicos ${ }^{8,9}$.

Adicionalmente, a suspensão do uso das mesmas por um determinado período de tempo pode reduzir a inflamação e iniciar a remissão do processo patológico ${ }^{10}$.

Devido ao grande número de reabilitações protéticas realizadas diariamente e à grande prevalência de lesões bucais causadas por microrganismos relacionadas, o objetivo deste estudo foi identificar a prevalência de lesões bucais microbianas em uma população específica.

\section{MATERIAL E MÉTODO}

Trata-se de um estudo transversal, quantitativo e descritivo realizado em uma Unidade de Saúde da Família na cidade de Jequié na Bahia. A população de estudo consistiu de 110 usuários de próteses dentárias parciais e/ou totais, removíveis e/ou fixas residentes na área de abrangência da unidade e que aceitaram participar do estudo sob consentimento livre e esclarecido. A coleta de dados foi realizada por meio de exame clínico com luvas, espátula de madeira e auxílio de uma lanterna de cabeça - para melhor visualização da cavidade bucal - no domicílio do participante.

O exame foi realizado por apenas um examinador (o pesquisador) e não houve secagem da cavidade bucal. Um formulário específico (Apêndice B) foi desenvolvido levando-se em consideração as variáveis: sexo, faixa etária, tipo de prótese, tempo de uso de prótese e tempo de uso da prótese atual, métodos de higienização bucal e da prótese bem como frequência de higienização e hábitos deletérios como, por exemplo, o uso noturno do aparato, consumo e frequência do consumo de bebidas alcoólicas e fumo. Após a coleta dos dados foi realizada orientação de higiene bucal e da prótese e quanto aos cuidados que devem ser tomados para a longevidade das peças protéticas. Os dados coletados foram tabulados em planilha do Microsoft Excel 2010 e analisados através de técnicas de estatística descritiva.

Este estudo foi aprovado pelo Comitê de Ética e Pesquisa da Universidade Estadual do Sudoeste da Bahia (UESB), Parecer $\mathrm{n}^{\mathrm{o}}$ : 148.203 sob CAAE: 06469112.1.0000.0055.

\section{RESULTADOS}

Dos 110 participantes da pesquisa $23(20,9 \%)$ eram do sexo masculino e $87(79,1 \%)$ eram do sexo feminino. Quanto à presença de lesões bucais causadas por microrganismos, $72(65,4 \%)$ usuários de prótese dentária não apresentaram nenhum tipo de acometimento patológico. A forma mais comum de lesão bucal foi a estomatite por prótese, sendo evidenciada em 30 indivíduos $(27,3 \%)$. Apenas $3(2,7 \%)$ participantes apresentaram queilite e $5(4,6 \%)$ uma associação de estomatite e queilite. $30,44 \%$ dos homens e $35,64 \%$ das mulheres apresentavam algum tipo de lesão.

A média de idade da população estudada foi 64 anos para homens e 60,6 anos para mulheres. Quanto ao tempo médio de uso de prótese, os valores encontrados foram 21,4 anos para os homens e 22,3 anos para as mulheres. Quanto ao tempo médio de uso da prótese 
atual, os homens usavam a mesma prótese por 6,9 anos e as mulheres por 7,9 anos. Para os participantes com estomatite, a média de uso da prótese atual foi de 12,7 anos. Já para os portadores de queilite essa média caiu para 6 anos, enquanto que os portadores de queilite e estomatite usaram a mesma prótese por 6,2 anos.
A Tabela 1 mostra a distribuição de usuários por diferentes tipos de prótese.

A Tabela 2 apresenta o número de usuários acometidos patologicamente segundo o tipo de lesão e prótese. No quadro, a letra 'a' representa a presença de estomatite, a letra 'b' a presença de queilite e as letras ' $a+b$ ' representam a associação das duas lesões.

Tabela 1. Distribuição do tipo de prótese entre usuários com diferenciação de gênero

\begin{tabular}{ccccccc}
\hline PT Sup & $\begin{array}{c}\text { PPR } \\
\text { Sup }\end{array}$ & $\begin{array}{c}\text { PT Sup } \\
\text { e Inf }\end{array}$ & $\begin{array}{c}\text { PT Sup } \\
\text { e PPR } \\
\text { Inf }\end{array}$ & $\begin{array}{c}\text { PPR } \\
\text { Sup e } \\
\text { Inf }\end{array}$ & $\begin{array}{c}\text { Total } \\
\text { geral }\end{array}$ \\
\hline $\mathbf{M}$ & 6 & 2 & 8 & 4 & 3 & 23 \\
\hline $\mathbf{F}$ & 30 & 6 & 34 & 7 & 10 & 87 \\
Total & 36 & 8 & 42 & 11 & 13 & 110 \\
\hline
\end{tabular}

Tabela 2. Número de usuários de acordo com a lesão e o tipo de prótese

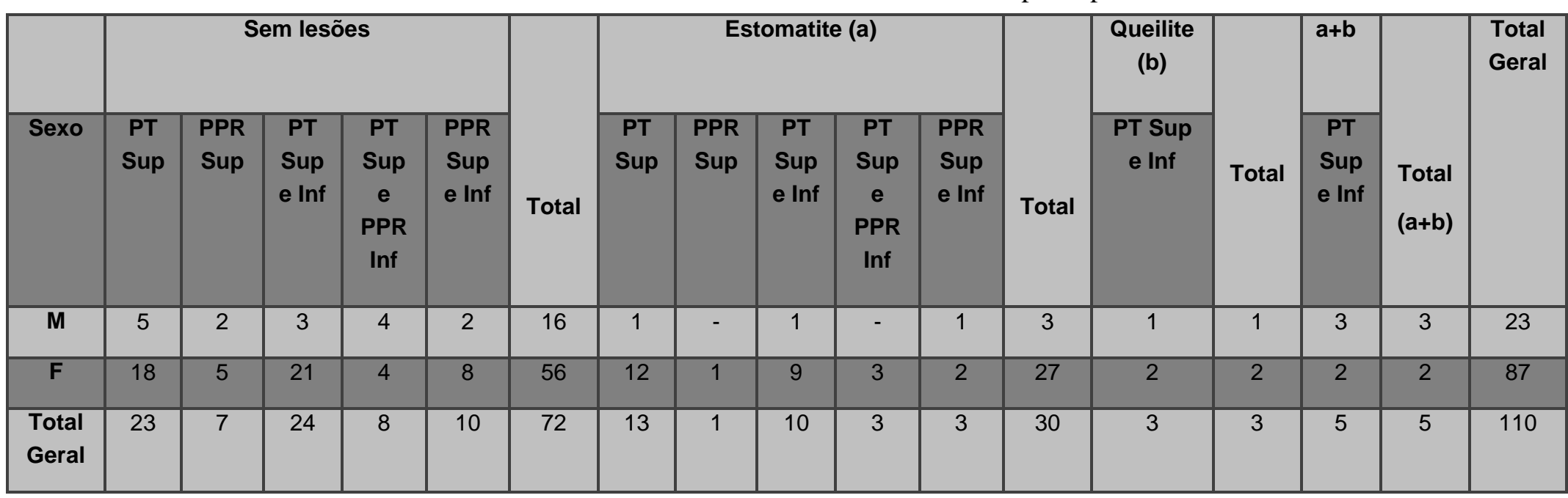

O método mais empregado na higienização das próteses foi a escovação com creme dental, porém alguns usuários relataram a utilização adicional de sabonete, sabão de coco, hipoclorito de sódio, vinagre e bicarbonato de sódio durante o processo de limpeza. Quanto à frequência, a maior parte dos participantes referiu higienizar as peças de 2 a 3 vezes por dia.

Do total da amostra, 46,4\% dos usuários de prótese possuíam higiene bucal deficiente. Destes, 54,9\% apresentavam algum tipo de lesão. 96\% dos usuários com boa higiene bucal não apresentavam nenhum sinal de lesão bucal. $83,3 \%$ dos que higienizavam a cavidade bucal e a(s) prótese(s) apenas 1 vez ao dia, 38,9\% dos que higienizavam 2 vezes e 27,9\% dos que faziam a higienização 3 vezes foram acometidos patologicamente. Foi detectada lesão em $39,2 \%$ dos $71,9 \%$ dos participantes que relataram fazer uso contínuo da peça protética, inclusive durante o sono. Não se observou diferença significativa entre sexos para essas duas análises.

Apenas 10 participantes são fumantes ativos, sendo 8 mulheres e 2 homens. Todos fumam há mais de 10 anos. Desses, 3 desenvolveram algum tipo de lesão bucal. Dentre os que nunca fumaram (42 indivíduos), 14 apresentaram lesão. Dos 58 indivíduos ex-fumantes, 20 eram portadores de alguma lesão - todos possuíam estomatite e dois deles apresentavam também queilite.

Em relação ao consumo de álcool, 90 pessoas nunca beberam e dessas 32 (35,5\%) tinham lesão - 2 queilite, 3 estomatite + queilite e 27 somente estomatite. Dos 20 participantes que bebem, 6 (30\%) apresentaram lesão -3 estomatite, 1 queilite e 2 estomatite + queilite.

\section{DISCUSSÃO}

O objetivo dos tratamentos reabilitadores protéticos é devolver função, estética e qualidade de vida ao paciente ${ }^{10}$. No entanto, a reconstituição do sistema estomatognático e cobertura da mucosa mastigatória com uma prótese representa uma agressão mecânica não fisiológica para o tecido bucal. Quando os efeitos de pressão, tração e empuxo produzidos pela prótese são de grande intensidade supõe-se um estímulo deletério que rompe o equilíbrio e conduz-se a uma irritação do tecido, seguida de suas consequentes reações inflamatórias ${ }^{11}$.

Neste estudo avaliou-se a prevalência de lesões orais relacionadas ao uso de prótese em 110 indivíduos. A maior parte da amostra $(79,1 \%)$ era do sexo feminino. Um maior número de mulheres também foi encontrado nos estudos de Coelho et $\mathrm{al}^{6}$, Bomfim et $\mathrm{al}^{10}$ e Maciel et $\mathrm{al}^{12}$. 
Alguns estudos como os de Bomfim et $a^{10}$, Maciel et $\mathrm{al}^{12}$ e Mujica et $\mathrm{al}^{13}$ relataram uma maior prevalência de lesões bucais em mulheres enquanto Patil et $\mathrm{al}^{14}$ relataram uma leve preponderância para o sexo masculino. No entanto, nesta pesquisa, apesar da casuística de mulheres ser muito maior em relação aos homens, os resultados mostraram que não houve uma diferença tão significativa entre os sexos quanto à presença de lesão $(30,44 \%$ sexo masculino e 35,64\% sexo feminino). Fato este também evidenciado por Ercalik-Yalcinkaya, Ozcan ${ }^{15}$.

Segundo Coelho et $\mathrm{al}^{6}$ e Pentenero et $\mathrm{al}^{16}$ as lesões orais estão associadas com maior frequência ao uso de próteses devido ao fato de que as próteses removíveis são, muitas vezes, causadoras de injúrias na mucosa bucal. Considerando-se o fato de que $65,4 \%$ dos usuários de prótese participantes deste estudo não apresentavam outro acometimento patológico na cavidade bucal, podese inferir que o uso de próteses dentárias somente não é causador do desenvolvimento de lesões orais. Maior susceptibilidade conferida pela idade, infecção por Candida, higiene deficiente da boca e das próteses são alguns dos fatores de risco citados na literatura ${ }^{5,6,17}$.

Jainkittivong et $\mathrm{al}^{18}$ e Patil et $\mathrm{al}^{14}$ afirmam que alterações fisiológicas e nutricionais sistêmicas, mudanças no ambiente bucal, higiene bucal deficiente, hábitos e uso de medicamentos associados à idade aumentam a prevalência de patologias orais em usuários de próteses dentárias.

Sabe-se que à medida que aumenta o tempo de uso da prótese maior a chance de se desenvolver lesões nos tecidos moles ${ }^{12,19}$. O tempo de uso de prótese é um grande contribuinte para estomatite protética $^{15}$, por exemplo.

A estomatite refere-se à infecção dos tecidos moles relacionados ao uso de próteses dentárias ${ }^{9}$ e é uma das lesões mais frequentes em pacientes reabilitados proteticamente ${ }^{5,8}$.

As lesões orais evidenciadas nesta pesquisa foram a estomatite protética $(27,3 \%)$ e a queilite angular $(2,7 \%)$. Estes resultados corroboram os achados de vários outros pesquisadores como Bomfim et $\mathrm{al}^{10}$ (44,6\% estomatite e $12,7 \%$ queilite angular); Maciel et $\mathrm{al}^{12}$ - $(78 \%$ das lesões eram estomatite e $4,9 \%$ queilite angular); Mujica et $\mathrm{al}^{13}$ - (18\% estomatite e $5 \%$ queilite); Ferreira et $\mathrm{al}^{17}$ - $\left(15,2 \%\right.$ estomatite); Espinoza et $\mathrm{al}^{19}$ (22,3\% estomatite) e Turker et $\mathrm{al}^{20}$ - (22,6\% estomatite) onde a estomatite foi a lesão mais prevalente e a queilite angular esteve entre as lesões mais encontradas.

Na população estudada, o tempo de uso de prótese variou de 21,4 anos para os homens e 22,3 anos para as mulheres.. Esse valor máximo foi o maior registrado em comparação a outros estudos ${ }^{6,10}$. Apesar dos homens usarem peças protéticas por um tempo médio maior que as mulheres, quanto ao quesito tempo de uso da prótese atual houve uma inversão onde as mulheres usavam a mesma prótese 1 ano a mais que os homens (7.9 anos de tempo de uso das mulheres contra 6.9 anos para os homens). Ferreira et al $^{17}$ afirmam que idosos usuários de prótese tendem a ser mais resistentes à substituição de peças protéticas antigas usando assim a mesma prótese por mais tempo.

Turker et $\mathrm{al}^{20}$ dizem que indivíduos que usam suas próteses por um tempo significativamente maior que o recomendado estão mais susceptíveis a desenvolver lesões orais. No presente estudo identificou-se que participantes que apresentavam estomatite usavam suas próteses por um período médio de 12,7 anos.

Além do tempo de uso, a higienização da boca e das próteses é de fundamental importância para a saúde bucal e prevenção de patologias. Evren et $\mathrm{al}^{21}$ relatam que estudos têm estabelecido uma associação entre a falta de programas preventivos, a falta de higienização adequada dos aparatos protéticos e a falha na remoção noturna das dentaduras com o desenvolvimento de lesões bucais.

Devido a importância da realização diária dos procedimentos de higiene para a manutenção da saúde bucal e do corpo e uma maior longevidade dos tratamentos reabilitadores, é essencial que os pacientes/usuários de próteses sejam alertados pelos profissionais de saúde, especialmente os dentistas, sobre a necessidade da adequada higienização das peças protéticas e das visitas periódicas ao dentista. Assim, há uma maior probabilidade dos tratamentos serem mais eficazes a partir do acompanhamento e intervenção profissional no que diz respeito à instrução do paciente, diagnósticos precoces e manutenção dos aparatos protéticos evitando-se maiores danos à saúde ${ }^{22}$. Todos os participantes da pesquisa afirmaram realizar a higiene tanto da boca quanto das próteses de 1 a 3 vezes por dia. Os resultados apontam menor ocorrência de desenvolvimento de lesões bucais quando a frequência de higienização é superior a 2 vezes por dia.

Quando questionados à respeito do método escolhido para higienização, 100\% da amostra declarou realizar escovação. $\mathrm{O}$ creme dental foi o produto mais utilizado durante esse processo, porém produtos como hipoclorito de sódio, sabonete, sabão de coco, vinagre e bicarbonato de sódio também foram citados como meios adjuvantes na limpeza das próteses. Coelho et $\mathrm{al}^{6}$, Bomfim et $\mathrm{al}^{10}$, Ercalik-Yalcinkaya, Ozcan ${ }^{15}$, Evren et $\mathrm{al}^{21}$ e Baran et $\mathrm{al}^{22}$ também relataram a utilização de tais produtos pelos sujeitos da pesquisa. Além da intervenção mecânica da escovação, Coelho et al $^{6}$ ainda citam em seu estudo a utilização de peróxido de hidrogênio, água fervida, arnica e álcool no processo de limpeza das próteses ilustrando a diversidade de produtos que são utilizados pela população.

Acredita-se que escovação com dentifrício é o método mais empregado na higienização por ser eficaz na remoção mecânica do biofilme bacteriano da 
superfície dentária e das próteses. No entanto, ErcalikYalcinkaya, Ozcan ${ }^{15}$ e Pellizzaro et al $^{23}$ alertam para o fato de que se por um lado a escovação remove a placa bacteriana aderida à superfície das próteses, por outro a ação abrasiva dos dentifrícios danifica essa superfície aumentando a rugosidade da peça e favorecendo a adesão e colonização de fungos.

Ercalik-Yalcinkaya, Ozcan ${ }^{15}$ dizem ainda que a desinfecção química das próteses pode degradar o polimetilmetacrilato - material utilizado na confecção das próteses - facilitando a colonização por microorganismos principalmente do gênero Candida. Por este motivo, os autores recomendam o emprego de produtos menos ou não abrasivos no momento da limpeza das peças protéticas e a imersão das mesmas em água quando fora da cavidade bucal a fim de evitar a perda e/ou alteração das propriedades dos materiais componentes das próteses ${ }^{15,23}$.

Apesar dos relatos sobre higienização, quase metade $(46,4 \%)$ dos participantes deste estudo apresentavam higiene bucal deficiente. Valor este muito próximo ao encontrado por Baran et $\mathrm{al}^{22}$ onde $42,9 \%$ dos participantes do estudo apresentaram sua higiene bucal como pobre/deficiente e por Bomfim et $\mathrm{al}^{10}$ onde $53,1 \%$ dos participantes tinham má higiene bucal.

Nesta pesquisa evidenciou-se que quase $40 \%$ dos indivíduos que faziam uso contínuo da prótese possuíam lesão. Ercalik-Yalcinkaya, Ozcan ${ }^{15}$ afirmam que encontra-se na literatura indícios de que o uso noturno de próteses é um fator desencadeante para alguns tipos de lesões orais como por exemplo estomatite protética e mostram que em seu estudo essa correlação foi positiva. Para os autores, a diminuição da capacidade protetora da saliva e da resistência da mucosa bucal contra agressões externas influenciada pelo uso contínuo de peças protéticas pode ser uma explicação para a maior susceptibilidade ao desenvolvimento das lesões microbianas na cavidade bucal. Ferreira et $\mathrm{al}^{17}$ complementam dizendo que o uso contínuo de próteses predispõe à irritação e infecção, todavia quando cessado o uso contínuo por duas semanas nota-se uma remissão espontânea do quadro irritativo/infeccioso da mucosa.

Além dos já mencionados, a literatura também elenca o estilo de vida e hábitos deletérios como fatores de risco para lesões orais. Álcool e tabaco, por exemplo, têm sido altamente relacionados ao desenvolvimento do câncer bucal e das alterações bucais menos graves ${ }^{15}$ como as lesões citadas neste estudo. Nesta pesquisa, constatou-se que todos os ex-fumantes, 3 dos 10 fumantes ativos e 6 dos 20 participantes que bebem apresentavam algum tipo de lesão.

Harris et al $^{24}$ afirmam que o consumo de álcool pode contribuir direta ou indiretamente para o aparecimento de lesões e diz que em seu estudo uma maior prevalência de lesões da mucosa bucal foi identificada nessa população de risco.
No estudo de Baran et $\mathrm{al}^{22}$, a comparação entre fumantes e ex-fumantes e indivíduos que nunca fumaram revelou que a prevalência de estomatite protética e úlceras traumáticas foi muito maior naqueles que fazem ou já fizeram uso do tabaco.

Cebeci et $\mathrm{al}^{25}$ observaram em seu estudo uma relação estatisticamente significante entre o tabaco e a ocorrência de lesão bucal, porém nenhuma relação relevante foi encontrada entre o consumo de álcool e lesões bucais. Pentenero et $\mathrm{al}^{16}$ reiteram que há uma ligação entre álcool e tabaco e lesões bucais

\section{CONCLUSÃO}

Há ainda um grande número de pessoas acometidas por lesões orais de fácil diagnóstico e intervenção. A frequência de higienização bucal e da prótese igual ou superior a 2 vezes por dia diminui o risco de desenvolvimento de patologias assim como o uso contínuo das peças protéticas elevam esse risco.

Os resultados ainda sugeriram relação positiva entre o uso de álcool e tabaco e o desenvolvimento de lesões. Por esta razão, é fundamental o acompanhamento profissional do indivíduo e a conscientização dos mesmos sobre as condutas necessárias para se alcançar e manter boa saúde e qualidade de vida.

Este papel deve ser reforçado nas universidades para que os cirurgiões-dentistas melhorem sua atuação para a qualidade da saúde bucal em pacientes usuários de próteses.

\section{REFERÊNCIAS}

1. Montero J, López JF, Galindo MP, Vicente P, Bravo M. Impact of prosthodontic status on oral wellbeing: a cross-sectional cohort study. J Oral Rehabil. 2009;36(8):592-600.

2. Hewlett SA, Yawson AE, Calys-Tagoe BNL, Naidoo N, Martey P, Chatterji S, et al. Edentulism and quality of life among older Ghanaian adults. BMC Oral Health. 2015;15:48. doi: 10.1186/s12903015-0034-6.

3. Felício CM, Cunha CC. Relações entre condições miofuncionais orais e adaptação de próteses totais. PCL. 2005;7(36):195-202.

4. Silva SO, Trentin MS, Linden MSS, Carli JP, Silveira Neto N, Luft LR. Saúde bucal do idoso institucionalizado em dois asilos de Passo Fundo RS. RGO. 2008;56(3):303-8.

5. Gendreau L, Loewy ZG. Epidemiology and etiology of denture stomatitis. J Prosthodont. 2011;20(4):25160.

6. Coelho CMP, Sousa YTCS, Daré AMZ. Denturerelated oral mucosal lesions in a Brazilian school of dentistry. J Oral Rehabil. 2004;31(2):135-9.

7. Neville BW, Damm DD, Allen CM, Bouquot JE. Patologia Oral e Maxilofacial. Rio de Janeiro: Guanabara Koogan; 2004. 
8. Tay LY, Jorge JH, Herrera DR, Campanha NH, Gomes BP, Santos FA. Evaluation of different treatment methods against denture stomatitis: a randomized clinical study. Oral Surg Oral Med Oral Pathol Oral Radiol. 2014;118(1):72-7.

9. Cadastro GA, Alegretti CE, Scabar LF, Barros FC, Armonia PL, Giovani EM. Terapia fotodinâmica no tratamento da queilite angular - relato de caso. Rev Inst Ciênc Saúde. 2008;26(4):482-6.

10. Bomfim IPR, Soares DG, Tavares GR, Santos RC, Araújo TP, Padilha WWN. Prevalência de lesões de mucosa bucal em pacientes portadores de prótese dentária. Pesq Bras Odontoped Clin Integr. 2008;8:117-121.

11. Sifontes LL, Martínez YS, Capote EP, Nápoles IJ, Gómez SMD. Comportamiento de la estomatitis subprótesis. Revista Archivo Médico de Camagüey. 2010;14(1):0-0.

12. Maciel SSSV, Souza RSV, Donato LMA, Albuquerque IGM, Donato LFA. Prevalência das lesões de tecidos moles causadas por próteses removíveis nos pacientes da Faculdade de Odontologia de Caruaru, PE, Brasil. Pesq Bras Odontoped Clin Integr. 2008;8:93-7.

13. Mujica V, Rivera H, Carrero M. Prevalence of oral soft tissue lesions in an elderly venezuelan population. Med Oral Patol Oral Cir Bucal. 2008;13(5):270-4.

14. Patil S, Doni B, Maheshwari S. Prevalence and Distribution of Oral Mucosal Lesions in a Geriatric Indian Population. Can Geriatr J. 2015;18(1):11-14.

15. Ercalik-Yalcinkaya S, Ozcan M. Mucosal Lesions of Prosthesis Wearers. J Prosthodont. 2014;24(4):271-8.

16. Pentenero M, Broccoletti R, Carbone M, Conrotto $\mathrm{D}$, Gandolfo S. Oral mucosal lesions in Turin. Oral Dis. 2008;14(4):356-66.

17. Ferreira RC, Magalhães CS, Moreira AN. Oral mucosal alterations among the institutionalized elderly in Brazil. Braz Oral Res. 2010;24(3):296302.

18. Jainkittivong A, Aneksuk V, Langlais RP. Oral mucosal lesions in denture wearers. Gerodontology. 2010;27(1):26-32.

19. Espinoza I, Rojas R, Aranda W, Gamonal J. Prevalence of oral mucosal lesions in elderly people in Santiago, Chile. J Oral Pathol Med. 2003;32(10):571-5.

20. Turker SB, Sener ID, Koçak A, Yilmaz S, Ozkan YK. Factors triggering the oral mucosal lesions by complete dentures. Arch Gerontol Geriatr. 2010;51(1):100-4.

21. Evren BA, Uludamar A, Iseri U, Ozkan YK. The association between socioeconomic status, oral hygiene practice, denture stomatitis and oral status in elderly people living different residential homes. Arch Gerontol Geriatr. 2011;53(3):252-7.
22. Baran I, Nalçaci R. Self-reported denture hygiene habits and oral tissue conditions of complete denture wearers. Arch Gerontol Geriatr. 2009;49(2):237-41.

23. Pellizzaro D, Polyzois G, Machado AL, Giampaolo ET, Sanitá PV, Vergani CE. Effectiveness of Mechanical Brushing with Different Denture Cleansing Agents in Reducing In Vitro Candida albicans Biofilm Viability. Braz Dent J. 2012;23(5):547-554.

24. Harris CK, Warnakulasuriya KAAS, Cooper DJ, Peters TJ, Gelbier S. Oral mucosal lesions in alcohol misusers. J Oral Pathol Med. 2004;33(5):253-9.

25. Cebeci ARİ, Gülşahı A, Kamburoğlu K, Orhan BK, Öztaş B. Prevalence and distribution of oral mucosal lesions in an adult turkish population. Med Oral Patol Oral Cir Bucal. 2009;14(6):272-7.

\section{CONFLITO DE INTERESSES}

Os autores declaram não haver conflitos de interesse.

\section{AUTOR PARA CORRESPONDÊNCIA}

Fabio Ornellas Prado

fop_@hotmail.com

Submetido em 14/06/2016 Aceito em 27/06/2016 